\title{
Novel coronavirus (COVID-19) in environmental engineering perspective
}

\author{
Mohammad Hadi Dehghani ${ }^{1,2} \cdot$ Sharmili Roy $^{3} \cdot$ Rama Rao Karri $^{4}$
}

Published online: 13 January 2022

○ The Author(s), under exclusive licence to Springer-Verlag GmbH Germany, part of Springer Nature 2022

Coronaviruses $(\mathrm{CoV})$ are a large family of viruses that can infect animals and humans. Many known coronaviruses caused a range of respiratory infections in humans, such as MERS-CoV and SARS-CoV. In rare cases, animal coronaviruses infect humans and then spread between them. SARS-CoV-2 is a new virus that has led to the outbreak of a respiratory disease known as novel coronavirus 2019 (COVID-19). The new virus is spreading rapidly around the world and devastated every country with many deaths. Evidences show that COVID-19 transmission occurs primarily between individuals through direct contact and then indirectly through close contact with infected persons via contaminated secretions or respiratory droplets of the infected person during coughing, sneezing, or while talking.

Healthcare centers, especially hospitals, are environments that can be the site of outbreaks of infectious diseases such as COVID-19. Therefore, if environmental and public health is not well monitored in these centers, staff and patients will face severe risks of viral and bacterial infections. Although healthcare professionals and other front-line workers are working round the clock to combat COVID-19, public participation is also essential to rapidly control the pandemic worldwide. Therefore, creating awareness among the public

Responsible Editor: Philippe Garrigues

Rama Rao Karri

drramarao.research@gmail.com; kramarao.iitd@gmail.com

1 Department of Environmental Health Engineering, School of Public Health, Tehran University of Medical Sciences, Tehran, Iran

2 Institute for Environmental Research, Center for Solid Waste Research, Tehran University of Medical Sciences, Tehran, Iran

3 Department of Medicine (Oncology), Stanford University, Stanford, CA 94305, USA

4 Faculty of Engineering, Universiti Teknologi Brunei, Gadong BE1410, Brunei Darussalam and sharing health protocols in order to control this epidemic are very important.

The protocols are rapidly changing, due to the severity prevailing at that situation, as well as more and more data from various sources are available. To address the issues and identify the source of spreading, researchers are constantly exploring the root cause and mitigating measures. Over the time, many research studies were conducted, and salient outcomes emerged.

To examine the measures that can be adopted at the time of a novel coronavirus outbreak to mitigate environmental contamination and impact on human health, the editors have edited a book, titled Environmental and Health Management of Novel Coronavirus Disease (COVID-19). Contributions come from virologists, microbiologists, public health professionals, environmental health managers, and policy makers. Further, to highlight how the coronavirus pandemic may have influenced the United Nations (UN) sustainable development goals (SDGs) and their implementation, research works related to COVID-19 and SDGs were compiled in an editorial book, COVID-19 and the Sustainable Development Goals.

There has been tremendous research carried out in the area of environmental engineering. However, there are limited single source in this perspective. To highlight the recent and relevant research studies in environmental engineering, this special issue (SI) is proposed and launched. This SI can be very effective in raising awareness to prevent and control COVID-19 through various environments such as air, wastewater, medical wastes, food products, and different surfaces. Research works related to COVID-19 in environmental engineering perspective are invited to this SI. Manuscripts covering research relevant to the following titles fit into the scope of this SI:

- Environmental transmission and spread routes of novel coronavirus 
- Epidemiological aspects and risk factors due to COVID19 pandemic

- COVID-19 effects on air quality and greenhouse gas emissions

- Statistical explorations and datasets of COVID-19 analysis to comprehend the disease spreading and death

- SARS-CoV-2 in wastewater and detection and removal methods of coronavirus in wastewater

- Presence of coronavirus in the solid waste environment and remedial approaches

- Transmission of COVID-19 via food products and associated hygiene safety

- Environmental disinfection technologies for the prevention of the spread of viruses

- Effect of the COVID-19 on access to renewable and sustainable energy management

- Mental, quality of life, and behavioral health aspects during the COVID-19 outbreak

- Effect of COVID-19 outbreak on educational status in the time of crisis

- Effect of COVID-19 on decent work, economic growth, and world trade

- Effect of remedial policies to contain the spread of the COVID-19 outbreak

- Global spread of COVID-19 and its influence on sustainable development goals

- Lessons learned from the COVID-19 pandemic, challenges, opportunities, and future perspective

In this special issue, we have received 49 articles which include short research and discussion articles, research articles, and review articles. All these articles are peer-reviewed by at least 2 independent reviewers, and only high-quality articles were considered for revision. After many rounds of revisions, only 18 high-quality articles were accepted amounting to $36 \%$ acceptance rate. In this, 4 are short research and discussion articles, and 9 are research articles and 5 review articles.

Acknowledgements We guest editors like to thank the editor-in-chief of the ESPR journal, the editorial assistant, and all the supporting staff for giving this opportunity.

Publisher's Note Springer Nature remains neutral with regard to jurisdictional claims in published maps and institutional affiliations.

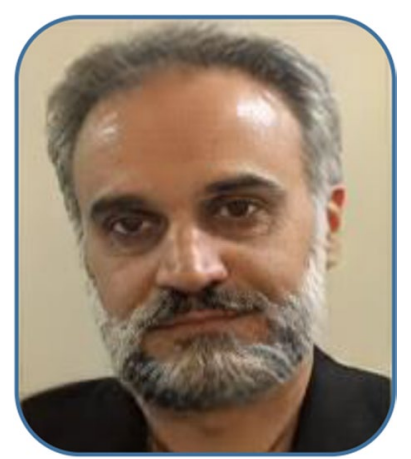

Prof. Dr. Mohammad Hadi Dehghani (PhD), He is a fulltime professor at the Tehran University of Medical Sciences (TUMS), School of Public Health, Department of Environmental Health Engineering, Tehran, Iran. His scientific research interests include environmental science. He is the author of various research studies published in national and international journals and conference proceedings and is head of several research projects at the TUMS. He has authored 12 books and more than 200 full papers published in peerreviewed journals. He is an editorial board member, guest editor, and reviewer in many internal and international journals and is a member of several international science committees around the world. He has a supervisor and advisor for $\mathrm{PhD}$ and MSc theses at the TUMS. He is currently also a member of the Iranian Association of Environmental Health and a member of the Institute for Environmental Research at the TUMS. He is an editor for 6 Elsevier edited books: (1) Soft computing techniques in solid waste and wastewater management, (2) Green technologies for the de-fluoridation of water, (3) Environmental and health management of novel coronavirus disease (COVID-19), (4) Pesticides remediation technologies from water and wastewater: Health effects and environmental remediation, (5) COVID-19 and sustainable development goals, and (6) Sustainable materials for sensing and remediation of noxious pollutants.

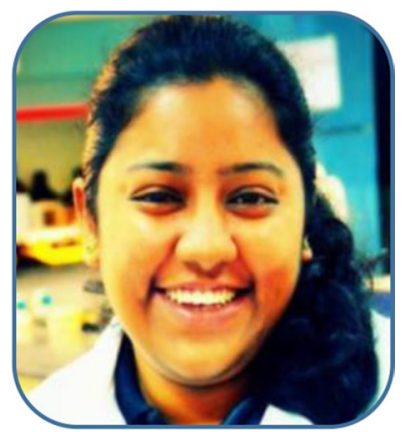

Dr. Sharmili Roy (PhD), She is currently pursuing her second post-doctoral research in oncology /medical research at the Division of Oncology, School of Medicine, Stanford University, California, USA. She earned her $\mathrm{PhD}$ from the Universiti of Brunei Darussalam, Brunei, under the supervision of Prof. Minhaz Uddin Ahmed. Before joining Stanford University, she worked for her first post-doctoral training at the Indian Institute of Technology Guwahati, India, under the supervision of Prof. Pranjal Chandra. Her current research is focused on the development of next-generation sequencing technology (NGS) with various clinical samples, especially with many kinds of cancer DNA samples, molecular diagnostics on cancer, and DNA digital data storage processes. She has expertized on point-of-care biosensor technology development based on loop-mediated isothermal amplification (LAMP) during her $\mathrm{PhD}$ tenure. She has published several high-impact factor research papers, 3 US patents, 4 book chapters in Biosensors and BioelectronicsAnalytical MethodsACS sensorsFood ControlFood chemistryElectro-analysis, etc. She along with Prof. Chandra has published a book titled, Diagnostic Strategies for Coronavirus (COVID-19) and other Coronaviruses Infections in Springer, May 2020. She is co-editor for 2 ongoing Elsevier edited books: (1) Environmental and Health Management of Novel Coronavirus Disease (COVID-19) and (2) COVID-19 and sustainable development goals. 


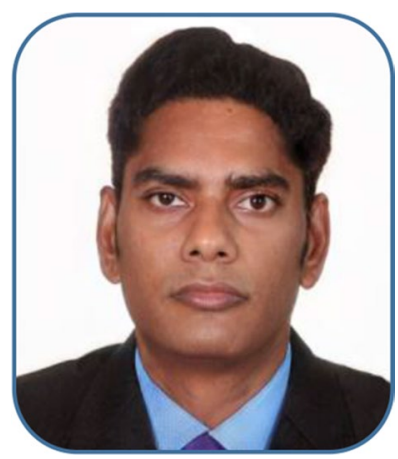

Dr. Rama Rao Karri (PhD), $\mathrm{He}$ is a professor (Sr. Asst) in the Faculty of Engineering, Universiti Teknologi Brunei, Brunei Darussalam. He has a $\mathrm{PhD}$ from the Indian Institute of Technology (IIT) Delhi and Masters from IIT Kanpur in Chemical Engineering. He has worked as a postdoctoral research fellow at NUS, Singapore, for about 6 years and has over 18 years of working experience in academics, industry, and research. He has experience working in multidisciplinary fields and has expertise in various evolutionary optimization techniques and process modelling. He has published 100+ research articles in reputed journals, book chapters, and conference proceedings with a combined impact factor of 312.2 and has an h-index of 23 (Scopus and Google Scholar). Among these publications, 50+ are Q1 and high IF journals. He is an editorial board member in 7 renowned journals and a peer-review member for more than 81 reputed journals and peer-reviewed more than 370 manuscripts. He is a recipient of Publons Peer Reviewer Award as top 1\% of global peer reviewers for Environment \& Ecology and Crossfield categories for the year 2019. He is also delegating as an advisory board member for many international conferences. He held a position as editor-in-chief (2019-2021) in the International Journal of Chemoinformatics and Chemical Engineering, IGI Global, USA. He is also an associate editor in Scientific Reports, Nature Group \& International Journal of Energy and Water Resources (IJEWR), Springer Inc. He is also a managing guest editor for Spl. Issues: (1) Magnetic nanocomposites and emerging applications, in Journal of Environmental Chemical Engineering (IF: 5.909); (2) Novel CoronaVirus (COVID-19) in Environmental Engineering Perspective, in Journal of Environmental Science and Pollution Research (IF: 4.223), Springer; and (3) Nanocomposites for the Sustainable Environment, in Applied Sciences Journal (IF: 2.679), MDPI. He along with his mentor, Prof. Venkateswarlu, is authoring an Elsevier bookOptimal state estimation for process monitoring, diagnosis and control. He is also co-editor and managing editor for 8 Elsevier, 1 Springer, and 1 CRC edited books. Recently, he has been listed in the 2021 world $2 \%$ scientists report compiled by Stanford University researchers. 